\title{
Diffusion and percolation in anisotropic random barrier models
}

\author{
Sebastian Bustingorry \\ Centro Atómico Bariloche, 8400 San Carlos de Bariloche, Río Negro, Argentina
}

(Dated: November 20, 2018)

\begin{abstract}
An anisotropic random barrier model is presented, in which the transition probabilities in different directions have different probability density functions. At low temperatures, the anisotropic longtime diffusion coefficients, obtained using an effective medium approximation, follow an Arrhenius temperature dependence, with the same activation energy for each direction. Such activation energy is related to the anisotropic percolation properties of the lattice, and can be analysed in terms of the critical percolation path approximation. The anisotropic effective medium approximation is shown to predict the correct percolation threshold for an anisotropic two-dimensional square lattice. In addition, results are compared with numerical simulations using a fast kinetic Monte Carlo algorithm.

PACS numbers: 05.40.-a, 05.60.Cd, 66.30.-h
\end{abstract}

\section{INTRODUCTION}

Diffusion in disordered media is an active field of research, due to its relevance in a wide variety of natural and industrial processes [1, 2, 3]. One of the traditional models for disorder is the random barrier model (RBM), which consists of equally energy minima separated by energy barriers, the height of which is randomly distributed according to a given probability density function (PDF). In this model, a particle moves from one minimum to another by performing thermally activated jumps.

In these systems, diffusion properties can be studied either in time or frequency variables. Several studies have been conducted of diffusion properties in the isotropic RBM both under unbiased [4, 5, 6, 7, 8, 9] and biased 10, 11 conditions. Following a frequency analysis (Refs. 4, 5] and references therein), the system may be characterized by a zero-frequency diffusion constant $D(s=0)$, and a characteristic frequency $s^{*}$, which marks the onset of frequency-dependent diffusion. $D(s=0)$ and $s^{*}$ follow Arrhenius laws with the same activation energy $E_{c}$. Analogously, from a time variable standpoint, it takes a time $t^{*} \sim s^{*-1}$ for a particle to reach a long-time diffusion regime in the RBM, characterized by a diffusion constant $D(t \rightarrow \infty) \equiv D(\omega=0)$ [ $]$. The activation energy $E_{c}$ depends on the percolation properties of the lattice and the PDF of the energy barriers. This dependence is simply achieved by the critical percolation path approximation (CPPA), as shown for isotropic problems 12, 13, 14].

In view of the diversity of systems in which diffusion takes place, the anisotropic generalization of diffusion problems has attracted considerable attention in the last years, both under unbiased [15, 16, 17, 18, 19, 20, 21] and biased 22, 23, 24] conditions. A few examples of anisotropic systems are porous reservoir rocks [3, 19, 25], layered semiconducting compounds 26], and superconductor cuprates [27]. When dealing with anisotropic conditions, diffusion properties are independently studied in the different relevant directions of the system. It was recently shown that, for a two-dimensional anisotropic bond percolation model, different activation energies are found in each direction 18.

In the present paper, a two-dimensional unbiased diffusion process is studied with each direction characterized by a different continuous PDF. The paper is organized as follows. In Sec. II the anisotropic RBM is introduced. In Sec. III the model is studied within the anisotropic effective medium approximation (EMA). These results are compared against Monte Carlo simulations, whose numerical details are given in Sec. IV Section $\nabla$ is devoted to a description of the CPPA ideas in anisotropic conditions, and in Sec. VI the concluding remarks of the present paper are presented.

\section{ANISOTROPIC RANDOM BARRIER MODEL}

Diffusion processes will be studied on a twodimensional square lattice with static disorder. Energy barriers are chosen from a given $\operatorname{PDF} \rho(E)$ at $t=0$ and are kept constant during the diffusion process. Possible jumps are only allowed between nearest neighbors. Once the energy barrier $E_{i j}$ between sites $i$ and $j$ is selected, the transition rates $\omega_{i j}$ from site $i$ to site $j$ are determined following an Arrhenius law

$$
\omega_{i j}=\frac{\omega_{0}}{z} e^{-\beta E_{i j}},
$$

where $\omega_{0}$ is the constant jump rate, $z=4$ is the coordination number, and $\beta=1 / k_{B} T$ is the inverse temperature, with $k_{B}$ being the Boltzmann constant. The energy $E_{i j}$ characterizes the bond joining sites $i$ and $j$, therefore $E_{i j}=E_{j i}$, and the forward $(i \rightarrow j)$ and backward $(j \rightarrow i)$ jumps have the same transition rate.

In order to introduce the anisotropic character of the system, the $E_{i j}$ energies are selected from different PDFs, depending on the orientation of the bond joining sites $i$ and $j$. Let 1 and 2 be the main directions of the square lattice, the key idea is to introduce $\rho_{1}\left(E_{1}\right)$ and $\rho_{2}\left(E_{2}\right)$ instead of a single PDF $\rho(E)$. The model is characterized 


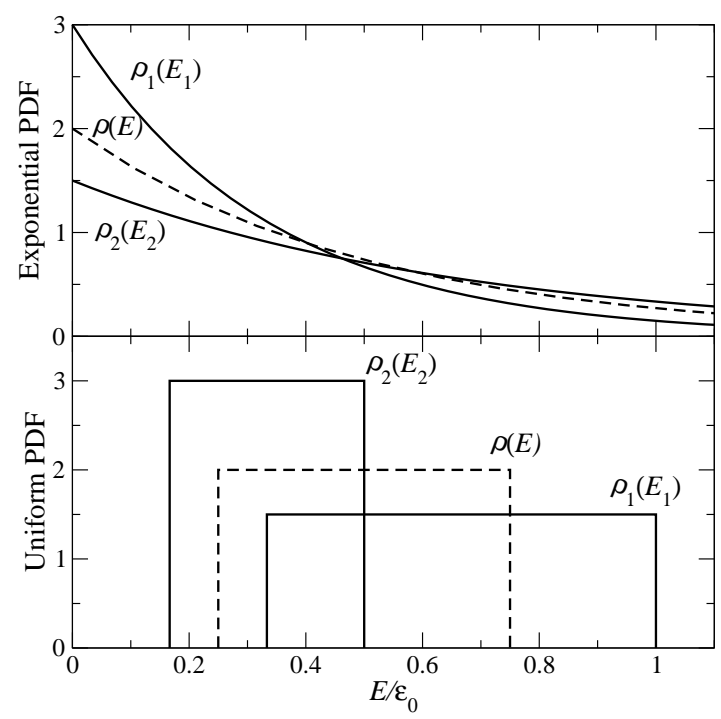

FIG. 1: Exponential (upper panel) and uniform (lower panel) probability density functions. Solid lines represent the anisotropic cases $(\alpha=2)$, and dashed lines the isotropic cases.

by anisotropy $\alpha=\epsilon_{1} / \epsilon_{2}$ and global mean energy $\epsilon=\left(\epsilon_{1}+\right.$ $\left.\epsilon_{2}\right) / 2$. The mean energies in each direction, $\epsilon_{1}$ and $\epsilon_{2}$, are thus represented by $\epsilon_{1}=2 \alpha \epsilon /(\alpha+1)$ and $\epsilon_{2}=2 \epsilon /(\alpha+1)$. In the present work, a constant value of $\epsilon=0.5 \epsilon_{0}$ is adopted, where $\epsilon_{0}$ sets the unit of energy, and the effects of having $\alpha \neq 1$ are studied. Two different anisotropic distributions will be considered: a) an exponential PDF

$$
\begin{array}{ll}
\rho_{1}\left(E_{1}\right)=\frac{1}{\epsilon_{1}} e^{-E_{1} / \epsilon_{1}}, & E_{1} \in[0, \infty), \\
\rho_{2}\left(E_{2}\right)=\frac{1}{\epsilon_{2}} e^{-E_{2} / \epsilon_{2}}, & E_{2} \in[0, \infty),
\end{array}
$$

and b) a uniform PDF

$$
\begin{array}{ll}
\rho_{1}\left(E_{1}\right)=\frac{1}{2 \delta_{1} \epsilon_{1}}, & E_{1} \in\left[\left(1-\delta_{1}\right) \epsilon_{1},\left(1+\delta_{1}\right) \epsilon_{1}\right], \\
\rho_{2}\left(E_{2}\right)=\frac{1}{2 \delta_{2} \epsilon_{2}}, & E_{2} \in\left[\left(1-\delta_{2}\right) \epsilon_{2},\left(1+\delta_{2}\right) \epsilon_{2}\right],
\end{array}
$$

where $\delta_{1}$ and $\delta_{2}$ serve to control different distribution widths in each direction. This uniform PDF represents the most general anisotropic extension of the isotropic uniform PDF used in Ref. [8] to study diffusion in RBM. In the following, and for the sake of simplicity, the widths of the uniform PDF will be $\delta_{1}=\delta_{2}=0.5$. Figure 1 shows the exponential and uniform PDFs for $\alpha=1$ and $\alpha=2$.

\section{ANISOTROPIC EMA: LOW TEMPERATURE PREDICTIONS}

The EMA self-consistent conditions provide a method for obtaining the diffusion coefficients for a given disor- dered medium. Usually, these equations must be numerically solved, except for some simple cases. It is showed in this section that for the low temperature limit, some analytical predictions may be obtained within the RBM.

\section{A. Self-consistent conditions}

Many authors 28, 29, 30, 31] have derived the EMA that allows to obtain effective diffusion coefficients. The approach considers one impure bond of the disordered lattice as embedded in an effective medium, mimicking the average surroundings. By imposing the averaged fluctuations to be zero, the self-consistent condition is derived for the transition rate of the effective medium. For an hypercubic $d$-dimensional isotropic lattice in the long-time limit, this condition reads [2]:

$$
\left\langle\frac{(\omega-\sigma)}{\omega+(d-1) \sigma}\right\rangle_{\nu(\omega)}=0,
$$

where $\omega$ is the transition rate of the impure bond distributed according to the $\operatorname{PDF} \nu(\omega), \sigma$ is the transition rate of the effective medium, and the brackets denote average over the $\operatorname{PDF} \nu(\omega)$. Solving the self-consistent condition for $\sigma$, the diffusion coefficient is obtained as $D=\sigma a^{2}$, where $a$ is the lattice constant.

The anisotropic extension of such formalism, where there exist $n$ different directions, leads to $n$ coupled equations that self-consistently solve for the $n$ different diffusion coefficients. In a two-dimensional square lattice, and for the long-time limit, these conditions are [15, 16]

$$
\left\langle\frac{\left(\omega_{m}-\sigma_{m}\right)}{\omega_{m}+\left(f_{m n}^{-1}-1\right) \sigma_{m}}\right\rangle_{\nu_{m}\left(\omega_{m}\right)}=0
$$

with

$$
f_{m n}=\frac{2}{\pi} \arctan \sqrt{\frac{\sigma_{m}}{\sigma_{n}}}
$$

and $m, n=1,2$ denoting the principal axes of the lattice. The effective transition rates $\sigma_{i}$ are related to the diffusion constants by $D_{i}=\sigma_{i} a^{2}$.

At high temperatures, the particle can easily overcome energy barriers, and eventually all diffusion constants approach the same value. In Fig. 2] the normalized diffusion coefficients at high temperatures for the twodimensional square lattice with an exponential PDF are plotted as functions of temperature, both under isotropic and anisotropic conditions. Lines represent the solutions of the EMA self-consistent conditions Eqs. (4) and (5), and symbols correspond to numerical simulations (see Sec. IV]. The figure shows that, for high temperatures, $D_{i} / a^{2} \omega_{0} \rightarrow 1 / z$. Analogous results are obtained using the uniform PDF. In the next Subsections the predictions of EMA for diffusion at low temperatures are considered. 


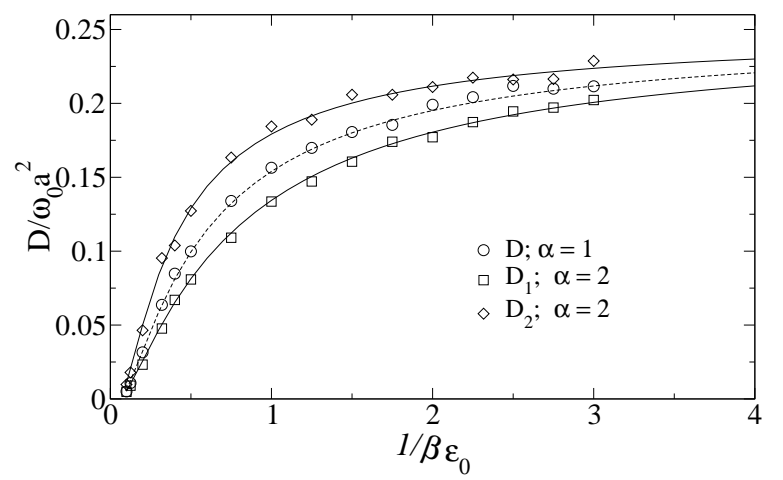

FIG. 2: Diffusion coefficients at high temperatures. Lines correspond to the EMA solution and symbols to numerical simulations. The isotropic case is represented by a dashed line and the anisotropic case $\alpha=2$ by continuous lines.

\section{B. Isotropic case}

For an isotropic hypercubic lattice in $d$ dimensions, Eq. (4) may be written as

$$
\left\langle\frac{(\omega+(d-1) \sigma-d \sigma)}{\omega+(d-1) \sigma}\right\rangle_{\nu(\omega)}=0 .
$$

By introducing the explicit dependence on energy $\omega=$ $\omega(E)$ given in Eq. (1), and transforming the transition rate average over $\nu(\omega)$ to an energy average over $\rho(E)$, Eq. (17) becomes

$$
\left\langle\frac{1}{\omega(E)+(d-1) \sigma}\right\rangle_{\rho(E)}=\frac{1}{d \sigma} .
$$

For $\beta \rightarrow \infty$ the transition rate $\omega(E)$ varies extremely rapidly, due to its exponential dependence. Therefore it is possible to define an energy value $E_{c}$ such that two possibilities arise: $\omega(E) \gg(d-1) \sigma$ for $E<E_{c}$, or $\omega(E) \ll(d-1) \sigma$ for $E>E_{c}$. The characteristic value $E_{c}$ can be therefore defined as

$$
\omega\left(E_{c}\right)=(d-1) \sigma
$$

For values of $E<E_{c}$, the left hand side of Eq. (8) vanishes. Alternatively, for $E>E_{c}$ the value $\omega(E)$ in the left hand side of Eq. (8) may be ignored. Taking these conditions into account, and averaging over $\rho(E)$, Eq. (8) for $\beta \rightarrow \infty$ becomes

$$
\frac{1}{d \sigma}=\int_{E_{c}}^{\infty} \frac{\rho(E)}{(d-1) \sigma} d E .
$$

Or, equivalently,

$$
\int_{0}^{E_{c}} \rho(E) d E=\frac{1}{d}
$$

In the EMA, the bond percolation threshold of the hypercubic lattice is given by $p_{c}^{E M A}=d^{-1}$ [28, 29, 30, 31]. Therefore, Eq. (11) is a condition over $E_{c}$ for each particular PDF $\rho(E)$, in terms of the percolation properties of the lattice. Indeed, combining this value of $E_{c}$ with Eq. (9), the EMA diffusion coefficient for isotropic $d$ dimensional hypercubic lattices at low temperatures is given by

$$
D=\frac{\omega_{0} a^{2}}{z(d-1)} e^{-\beta E_{c}}
$$

It is worth noting that $p_{c}^{E M A}=d^{-1}$ is only exact for $d=2$ [32], therefore Eq. (12) does not give the correct exponential behavior for $d=3$, and other approximations, such as CPPA, should be considered [7].

\section{Anisotropic two-dimensional case}

For the anisotropic two-dimensional case, Eq. (5) turns into two self-consistent conditions, with transition rate $\operatorname{PDFs} \nu_{1}\left(\omega_{1}\right)$ and $\nu_{2}\left(\omega_{2}\right)$, for each direction of the lattice. By introducing the energy dependence $\omega_{1}=\omega\left(E_{1}\right)$ and $\omega_{2}=\omega\left(E_{2}\right)$, the two self-consistent conditions read

$$
\begin{aligned}
& \left\langle\frac{1}{\omega\left(E_{1}\right)+\left(f_{12}^{-1}-1\right) \sigma_{1}}\right\rangle_{\rho_{1}\left(E_{1}\right)}=\frac{f_{12}}{\sigma_{1}}, \\
& \left\langle\frac{1}{\omega\left(E_{2}\right)+\left(f_{21}^{-1}-1\right) \sigma_{2}}\right\rangle_{\rho_{2}\left(E_{2}\right)}=\frac{f_{21}}{\sigma_{2}} .
\end{aligned}
$$

Again, the PDFs change abruptly for $\beta \rightarrow \infty$, and a parameter $E_{c}$ can be defined as in the isotropic case. However, $E_{c}$ is expected to be characteristic of the underlying energy landscape, so the diffusion coefficients in each direction are expected to be governed by a single $E_{c}$. For the anisotropic case, an energy $E_{c}$ will be defined separating two limiting conditions simultaneously: $\omega\left(E_{1}\right) \ll\left(f_{12}^{-1}-1\right) \sigma_{1}$ and $\omega\left(E_{2}\right) \ll\left(f_{21}^{-1}-1\right) \sigma_{2}$ for $E_{1}$ and $E_{2}$ larger than $E_{c}$, and $\omega\left(E_{1}\right) \gg\left(f_{12}^{-1}-1\right) \sigma_{1}$ and $\omega\left(E_{2}\right) \gg\left(f_{21}^{-1}-1\right) \sigma_{2}$ for $E_{1}$ and $E_{2}$ smaller than $E_{c}$. Thus, $E_{c}$ must verify two simultaneous conditions:

$$
\begin{gathered}
\omega\left(E_{c}\right)=\left(f_{12}^{-1}-1\right) \sigma_{1}, \\
\omega\left(E_{c}\right)=\left(f_{21}^{-1}-1\right) \sigma_{2} .
\end{gathered}
$$

In this way, a set of equations analogous to Eq. (11) are obtained,

$$
\begin{aligned}
& \int_{0}^{E_{c}} \rho_{1}\left(E_{1}\right) d E_{1}=f_{12}, \\
& \int_{0}^{E_{c}} \rho_{2}\left(E_{2}\right) d E_{2}=f_{21} .
\end{aligned}
$$


By adding Eqs. (15), using Eq. (6) and trigonometric relations, an expression is arrived at,

$$
\int_{0}^{E_{c}} \rho_{1}\left(E_{1}\right) d E_{1}+\int_{0}^{E_{c}} \rho_{2}\left(E_{2}\right) d E_{2}=1,
$$

which gives the activation energy $E_{c}$ as a function of the anisotropy $\alpha$. Moreover, by replacing the expressions in Eqs. (15) for $f_{12}$ and $f_{21}$ in Eqs. (14), and solving for $\sigma_{1}$ and $\sigma_{2}$, the corresponding anisotropic diffusion coefficients are obtained,

$$
\begin{aligned}
D_{1}= & \frac{\int_{0}^{E_{c}} \rho_{1}\left(E_{1}\right) d E_{1}}{\int_{0}^{E_{c}} \rho_{2}\left(E_{2}\right) d E_{2}} \frac{\omega_{0} a^{2}}{z} e^{-\beta E_{c}}, \\
D_{2}= & \frac{\int_{0}^{E_{c}} \rho_{2}\left(E_{2}\right) d E_{2}}{\int_{0}^{E_{c}} \rho_{1}\left(E_{1}\right) d E_{1}} \frac{\omega_{0} a^{2}}{z} e^{-\beta E_{c}} .
\end{aligned}
$$

The isotropic result Eq. (12) is obviously recovered by setting $\rho_{1}=\rho_{2}$.

Figures 3 and 4 show Arrhenius plots of the anisotropic diffusion coefficients at low temperatures, corresponding to the exponential and uniform PDFs, respectively. The solution of EMA self-consistent conditions Eqs. (4) and (5.) are represented with dashed an continuous lines, for the isotropic and anisotropic $\alpha=2$ cases, respectively. Dotted lines represent the prediction of EMA for the low temperature limit, Eqs. (17). Symbols are the results of numerical simulations, as described in the next section. These figures show that the diffusion coefficients in each direction follow Arrhenius laws with the same activation energy. Even though simulation data at lower temperatures are needed, the agreement with the low temperature anisotropic diffusion coefficients is better for a uniform PDF than for an exponential PDF.

\section{NUMERICAL SIMULATIONS}

Monte Carlo simulations were performed to obtain long-time diffusion coefficients for comparison with anisotropic EMA predictions. The energy landscape was selected from the corresponding PDF at $t=0$ and kept fixed during the diffusion process. At $t=0$ a particle was assigned to a random initial site $i$. Different Monte Carlo algorithms may be used at this point and two possibilities were considered: standard Monte Carlo (SMC) 8] and a fast kinetic Monte Carlo (FKMC) 33 scheme. A brief description of these methods is given in the following.

In SMC, the particle selects at random one of its nearest neighbors $j$ and tries to overcome the barrier between them in a time unit. A random number $\xi \in(0,1)$ is generated such that if $\xi<\omega_{i j}$ the jump is effective, otherwise

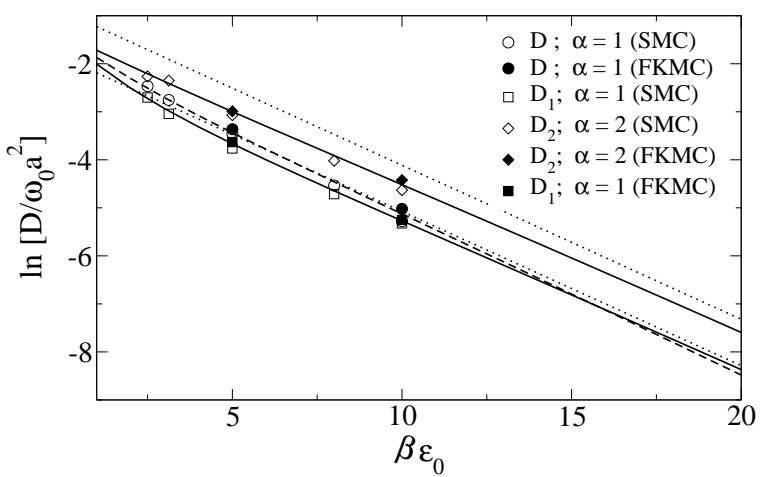

FIG. 3: Arrhenius plot of the diffusion coefficients for an exponential PDF. The solution of the self-consistent EMA conditions is represented with a dashed line for the isotropic case, and with continuous lines for the anisotropic $\alpha=2$ cases. Dotted lines represent the analytical EMA predictions, Eqs. (17), for low temperatures. Symbols correspond to SMC and FKMC simulations, as indicated.

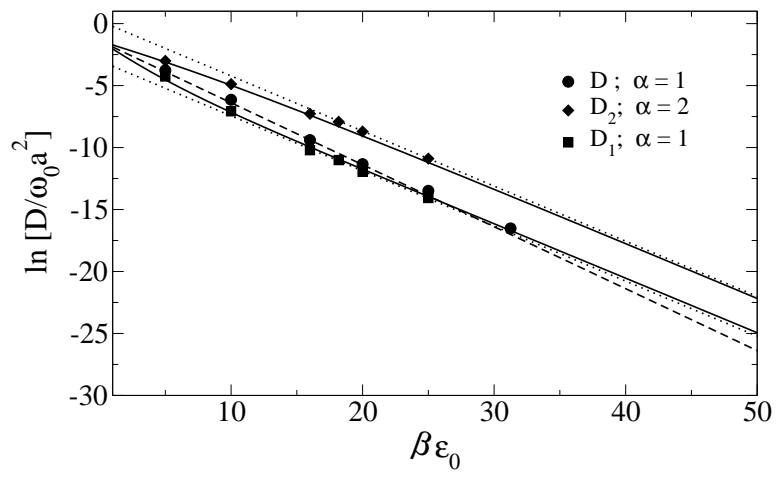

FIG. 4: Arrhenius plot of the diffusion coefficients for a uniform PDF. The solution of the self-consistent EMA conditions is represented with a dashed line for the isotropic case, and with continuous lines for the anisotropic $\alpha=2$ cases. Dotted lines represent the analytical EMA predictions, Eqs. [17), for low temperatures. Symbols correspond to FKMC simulations.

the particle stays at the initial site. In this process, one unit of time is used for every jump trial. Although SMC simulations proved to be very useful for studying diffusion processes, it was shown that it is not too appropriate for studying low temperature regimes $[5,[8,[33]$. At low temperatures, the transition rates decrease exponentially with increasing $\beta$, and the random number $\xi$ is, mostly, orders of magnitude greater than the transition rates, making the number of effective jumps (displacements) very small and the long-time diffusion regime difficult to reach.

In the FKMC 33] scheme, consider the particle in a site $i$ on a lattice with its $z$ nearest neighbors $j(j=$ 
$1, \ldots, z)$. The transition rates from $i$ to $j$ are denoted $\omega_{i j}$. The total transition rate $\omega_{i}$ from site $i$ is defined as:

$$
\omega_{i}=\sum_{j=1}^{z} \omega_{j i} .
$$

Instead of selecting the neighbor at random, as in SMC, a neighbor $k$ is selected for an effective jump given that

$$
\frac{1}{\omega_{i}} \sum_{j=1}^{k-1} \omega_{j i}<\xi_{1} \leq \frac{1}{\omega_{i}} \sum_{j=k}^{z} \omega_{j i},
$$

where $\xi_{1}$ is randomly uniformly distributed in $(0,1)$. The time variable $t$ is then increased in $t^{\prime}$, where $t^{\prime}$ is chosen from an exponential distribution with mean waiting time $\omega_{i}^{-1}$. Therefore,

$$
t^{\prime}=-\frac{1}{\omega_{i}} \ln \xi_{2},
$$

with $\xi_{2}$ randomly uniformly distributed in $(0,1)$. This procedure is repeated from site $k$ and so on. In the FKMC algorithm, each jump trial is effective, meaning that the particle always jumps to one of its neighbors, and the time elapsed in one jump is accordingly adjusted. Furthermore, the FKMC algorithm depends on the ratios $\omega_{i i} / \omega_{i}$ and consequently it is not as $\beta$ dependent as $\omega_{j i}$ 33. This algorithm allows to reach larger values of $\beta$ in simulations of the diffusion process.

Simulations were performed on $300 \times 300$ and $500 \times 500$ sites square lattices for the SMC and FKMC, respectively, with periodic boundary conditions. For each algorithm, the mean square displacements on each direction $\left\langle r_{1}^{2}(t)\right\rangle$ and $\left\langle r_{2}^{2}(t)\right\rangle$ were computed, averaging over between 2000 and 5000 realizations of the random walks. The long-time diffusion coefficients were defined through $\left\langle r_{1(2)}^{2}(t)\right\rangle=2 D_{1(2)} t$, and were obtained from the best linear fits to the long-time mean square displacements.

In Figs. 3 and 4 numerical simulations and EMA results are presented together. In Fig. 3] SMC simulations are plotted up to $\beta \epsilon_{0}=10$ and some FKMC simulation points are shown for comparison. Both algorithms coincide within the numerical precision. In Fig. 4, only FKMC results are presented up to a value $\beta \epsilon_{0}=30$. Monte Carlo simulations do not completely reach the asymptotic low temperatures behavior. However, numerical simulations and EMA seem to agree very well in the accessible temperature range.

\section{CRITICAL PERCOLATION PATH APPROXIMATION}

The idea of a percolation path governing diffusion at low temperatures was first developed in Ref. [12] and rigorously proved later [13, 14]. In this section, this idea will be briefly summarized and extended to anisotropic conditions.
At low temperatures the characteristic Arrhenius diffusion energy $E_{c}$ can be related to the bond percolation threshold of the lattice. Consider a random walk on a realization of the disorder energy landscape at a very low temperature. In order to overcome a barrier with an energy $E^{\prime}$, the particle spends a mean waiting time $t^{\prime} \sim \exp \left(\beta E^{\prime}\right)$. For short times, therefore, the particle can only move to sites which are connected by low energy barriers and is surrounded by a perimeter of higher energy. Roughly, at time $t^{\prime}$ the particle might jump barriers with $E \leq E^{\prime}$, and the probability to overcome this barriers is $\int_{0}^{E^{\prime}} \rho(E) d E$. For longer times, the particle could overcome the lowest barrier of the perimeter, and access a new region with a higher energy perimeter. These regions are non-compact in the sense that they may have inside barriers that belong to the perimeter barriers. Eventually, there exists a particular barrier of height $E_{c}$, beyond which the particle gains accesses to the whole system, through the corresponding percolation path of energies $E \leq E_{c}$. Thus, for an isotropic medium, $E_{c}$ is given by

$$
\int_{0}^{E_{c}} \rho(E) d E=p_{c},
$$

where $p_{c}$ is the bond percolation threshold of the system $\left(p_{c}=0.5\right.$ for the two dimensional isotropic square lattice [32]). It has been shown that $E_{c}$ is the highest energy barrier which the particle must overcome in order to gain full access to the percolation network. The long-time diffusion coefficient must therefore be $D \sim \exp \left(-\beta E_{c}\right)$, which is indeed the observed behavior of isotropic diffusion at low temperatures $[4,8]$.

The percolation threshold of a particular lattice, which is given by a point $p_{c}$ for isotropic percolation, becomes for anisotropic percolation a critical surface $\varphi\left(\left\{p_{i}\right\}\right)=0$ 34], where $\left\{p_{i}\right\}$ denotes the set of relevant occupation probabilities. For example, the percolation function is: $\varphi(p)=p-p_{c}$ for isotropic percolation, $\varphi\left(p_{1}, p_{2}\right)=$ $p_{1}+p_{2}-1$ for the square lattice, and $\varphi\left(p_{1}, p_{2}, p_{3}\right)=$ $p_{1}+p_{2}+p_{3}-p_{1} p_{2} p_{3}-1$ for the triangular lattice [34]. Furthermore, the critical surface implies a change in the morphology of the incipient percolation network.

In the anisotropic RBM context, the occupation probabilities of a bond with energy barrier $E$, i.e. accessibility condition of the bond, is given by the probability of $E$ being lower than the maximum accessible barrier. Therefore, the generalization of Eq. (21) to anisotropic conditions becomes

$$
\varphi\left(\left\{\int_{0}^{E_{c}} \rho_{i}\left(E_{i}\right) d E_{i}\right\}\right)=0 .
$$

Note that there exists just one energy $E_{c}$, which is the same for all directions, and gives full access to the whole anisotropic percolation network. For the anisotropic RBM on a square lattice, Eq. (22) becomes equal to the 


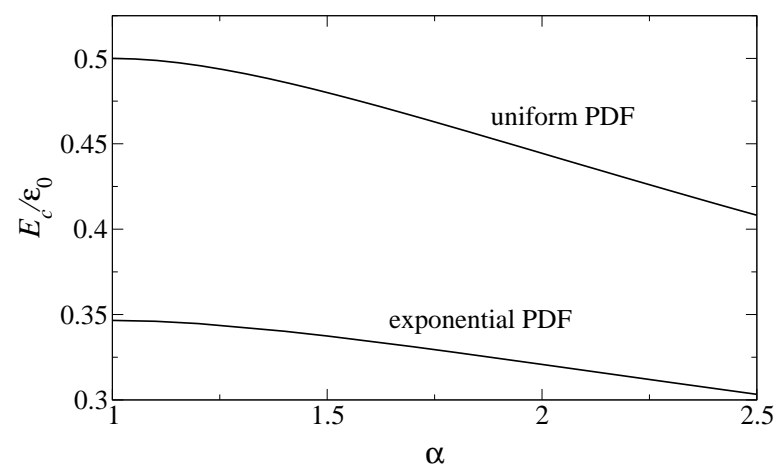

FIG. 5: Dependence of the activation energy $E_{c}$ on the anisotropic parameter $\alpha$ for the exponential and uniform PDFs studied here.

EMA prediction, Eq. (16). This means that EMA predicts the correct critical percolation surface $\varphi\left(p_{1}, p_{2}\right)=$ $p_{1}+p_{2}-1$ for anisotropic bond percolation in the square lattice [20, 21].

Figure 5 shows the effect of anisotropy $\alpha$ on $E_{c}(\alpha)$ for the energy distributions studied in the present model, and predicted both by CPPA Eq. (22), and EMA Eq. (16). For the exponential PDF, the condition for $E_{c}$ reads $\exp \left(-E_{c} / \epsilon_{1}\right)+\exp \left(-E_{c} / \epsilon_{2}\right)=1$, which was numerically solved. For the uniform PDF Eq. (16) gives $E_{c} / \epsilon_{0}=$ $2 \alpha /(\alpha+1)^{2}$.

\section{CONCLUDING REMARKS}

In this paper, diffusion properties were studied using an anisotropic RBM, with emphasis on the low temperature behavior and on percolation properties. Two kind of PDFs were used to characterize different directions of the lattice, namely, exponential and uniform PDFs. The anisotropic EMA was used to calculate the long-time diffusion properties for all temperatures, derived from the numerical solutions of the self-consistent conditions expressed in Eqs. (5). Furthermore, analytical expressions for the diffusion coefficients at low temperatures were obtained, Eq. (17), which show that diffusion in different directions follows Arrhenius laws with a same activation energy $E_{c}$. This should be compared with the thermally activated diffusion in anisotropic bond percolation lattices, in which different activation energies are found for each direction [18]. In the present model, only one activation energy is found due to the existence of an anisotropic percolation path of low energy barriers, which governs the diffusion process. Besides of giving the activation energy for diffusion, EMA predicts the exponential prefactor for diffusion and it dependence with the anisotropy of the disordered system.

The two Monte Carlo algorithms used here, namely, SMC and FKMC, show a very good agreement with the EMA predictions for the diffusion coefficients in the accessible temperature range. For a more extensive comparison with EMA, other algorithms should be used.

In the present paper, a connection was established between EMA and CPPA ideas, and EMA was shown to predict the correct activation energy for anisotropic diffusion in the square lattice. For other geometries and dimensions, it is expected that EMA will still predict an Arrhenius behavior, but with an activation energy that differs from that predicted by CPPA. This difference is due to the fact that CPPA uses the percolation threshold as a parameter, while EMA predicts its own percolation threshold. However, EMA is known to predict the correct percolation threshold only for the square lattice, even in anisotropic conditions 20, 21]. Concerning CPPA, corrections of the form $\beta^{y}$ become relevant for dimensions grater than two, but it is not clear which of both approximations, EMA or CPPA, give better results 7] and a systematic comparison turns necessary. Additional work on this direction is now under progress.

\section{ACKNOWLEDGMENTS}

The author want to thanks G. L. Insua for useful discussions. This work was financially supported by CONICET, Argentina.
[1] J. W. Haus and K. W. Kehr, Phys. Rep. 150, 263 (1987).

[2] J. P. Bouchaud and A. Georges, Phys. Rep. 195, 127 (1990).

[3] M. Sahimi, Flow and Transport in Porous Media and Fractured Rock (VCH, Weinheim, Germany, 1995).

[4] J. C. Dyre, Phys. Rev. B 49, 11709 (1994).

[5] J. C. Dyre and T. B. Schroder, Rev. Mod. Phys. 72, 873 (2000).

[6] I. Avramov, A. Milchev, and P. Argyrakis, Phys. Rev. E 47, 2303 (1993).

[7] H. Ambaye and K. W. Kehr, Phys. Rev. E 51, 5101 (1995).
[8] P. Argyrakis, A. Milchev, V. Pereyra, and K. W. Kehr, Phys. Rev. E 52, 3623 (1995).

[9] A. Hörner, A. Milchev, and P. Argyrakis, Phys. Rev. E 52, 3570 (1995).

[10] E. Arapaki, P. Argyrakis, I. Avramov, and A. Milchev, Phys. Rev. E 56, R29 (1997).

[11] I. Avramov, A. Milchev, E. Arapaki, and P. Argyrakis, Phys. Rev. E 58, 2788 (1998).

[12] V. Ambegaokar, B. I. Halperin, and J. S. Langer, Phys. Rev. B 4, 2612 (1971).

[13] S. Tyc and B. I. Halperin, Phys. Rev. B 39, 877 (1989).

[14] P. L. Doussal, Phys. Rev. B 39, 881 (1989). 
[15] P. E. Parris, Phys. Rev. B 36, 5437 (1987).

[16] E. R. Reyes, M. O. Cáceres, and P. A. Pury, Phys. Rev. B 61, 308 (2000).

[17] M. O. Cáceres and E. R. Reyes, Physica A 227, 277 (1996).

[18] S. Bustingorry and G. L. Insua, Phys. Rev. E 68, 012101 (2003).

[19] M. Saadatfar and M. Sahimi, Phys. Rev. E 65, 036116 (2002).

[20] J. Bernasconi, Phys. Rev. B 9, 4575 (1974).

[21] P. G. Toledo, H. T. Davis, and L. E. Scriven, Chem. Eng. Sci. 47, 391 (1992).

[22] S. Bustingorry, E. R. Reyes, and M. O. Cáceres, Phys. Rev. E 62, 7664 (2000).

[23] S. Bustingorry, M. O. Cáceres, and E. R. Reyes, Phys. Rev. B 65, 165205 (2002).

[24] S.-Y. Huang, X.-W. Zou, and Z.-Z. Jin, Phys. Rev. E 65, 052105 (2002).
[25] J. M. V. A. Koelman and A. de Kuijper, Physica A 247, 10 (1997).

[26] L. K. Gallos, A. N. Anagnostopoulos, and P. Argyrakis, Phys. Rev. B 50, 14643 (1994).

[27] H. Jhans, D. Kim, R. J. Rasmussen, and J. M. Honig, Phys. Rev. B 54, 11224 (1996).

[28] S. Alexander, J. Bernasconi, W. R. Schneider, and R. Orbach, Rev. Mod. Phys. 53, 175 (1981).

[29] T. Odagaki and M. Lax, Phys. Rev. B 24, 5284 (1981).

[30] I. Webman, Phys. Rev. Lett. 47, 1496 (1981).

[31] S. Summerfield, Solid State Commun. 39, 401 (1981).

[32] D. Stauffer and A. Aharony, Introduction to Percolation Theory (Taylor \& Francis, London, 1994).

[33] F. M. Bulnes, V. D. Pereyra, and J. L. Riccardo, Phys. Rev. E 58, 86 (1998).

[34] G. Grimmet, Percolation (Springer-Verlag, Berlin, 1990). 